\title{
Semantic Portrait Color Transfer with Internet Images
}

\author{
First Author • Second Author
}

Received: date / Accepted: date

\begin{abstract}
We present a novel color transfer method for portraits by exploring their high-level semantic information. Given a source portrait image, we first use Face ++ to search images with similar poses as the input from a database, and the user chooses one satisfactory image from the results as the target. The database consists of a collection of portrait images downloaded from the Internet, and each of them is manually segmented using image matting as a preprocessing step. Second, we extract portrait foregrounds from both source and target images. Third, the system extracts the semantic information, such as faces, eyes, eyebrows, lips, teeth, etc., from the extracted foreground of the source using image matting algorithms. After that, we perform color transfer between corresponding parts with the same semantic information. We get the final transferred result by seamlessly compositing different parts together using alpha blending. Experimental results show that our semantics-driven approach can generate better color transfer results for portraits than previous methods and provide users a new means to retouch their portraits.
\end{abstract}

Keywords color transfer · face detection · image matting

\section{Introduction}

Color transfer aims to keep the content of the source image as well as accurately apply the color style of the tar-

\section{F. Author}

first address

Tel.: +123-45-678910

Fax: $+123-45-678910$

E-mail: fauthor@example.com

S. Author

second address get image [1], so that the color styles of the source image and the target image are consistent. Nowadays, color transfer has been widely used in film post-production, video editing, artistic design, and image processing.

With the increasing number of mobile phones and camera devices, more and more people like to share their daily lives with photos. Many of these photos are lack of hierarchy, underexposure and low color saturation. To this end, some image processing software tools have been developed to retouch photos in order to make these photos look more exquisite and attractive. However, these software tools need special skills to use. Therefore, developing an efficient and easy-to-use image editing tool which can promote the attractiveness of portraits is demanding. We notice that there are lots of attractive and artistic portraits in the Internet. These portraits are usually taken by professional photographer using professional cameras. This motivates us to develop a new color transfer technique which can transfer the color style of a portrait by utilizing a database consists of a collection of portrait images downloaded from Internet.

Color transfer is a widely used tool in computer graphics and photography by performing statistical analysis in a carefully chosen color space $[1,6,7,9]$. They extract various color statistics from the source image and the target image, then establish specific mapping functions to make the color of both images consistent. In many cases, the low level statistical information is not sufficient to describe the content of the image, and certain areas may be incorrectly colored during the mapping process. To avoid such mistakes, some interactive methods are proposed $[15,17]$. In these methods, users can specify the color strokes, so that to ensure colors between different image regions can be properly converted. However, non-professional users may be diffi- 


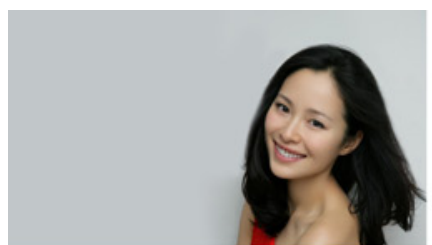

(a) Source Image

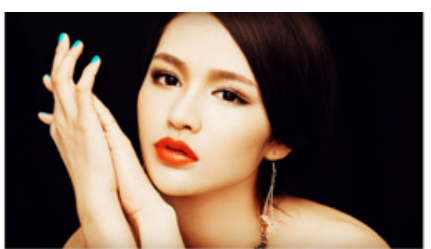

(b) Target Image

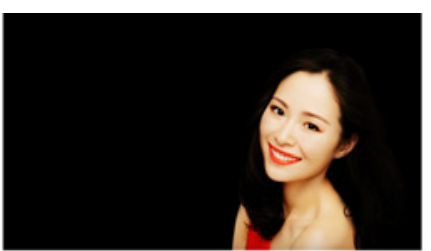

(c) Our Result

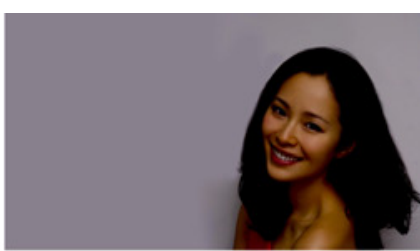

(d) [RAGS01]

Fig. 1 Our paper proposes a method based on facial semantic information to transfer the color style of an image. Experimental results show that our approach retains the contents of the source image, gains the spatial distribution of the target image and has the strong visual coherence in (c). We can see the color spatial distribution in the target image is mean to each pixel of each channel in $(\mathrm{d})$.

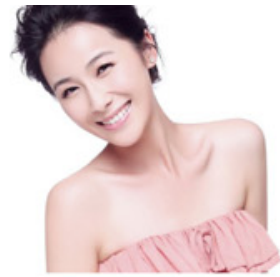

(a) Source Image

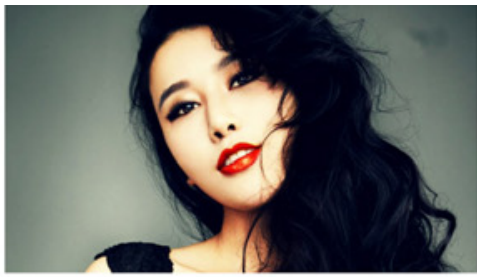

(b) Target Image

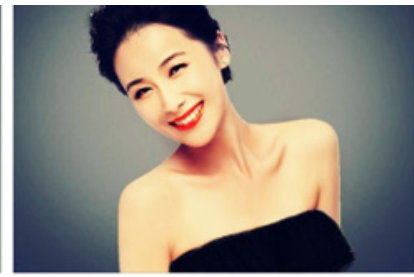

(c) Our Result

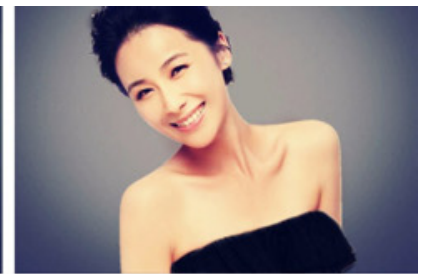

(d) $[$ AP10]

Fig. 2 Our method can help users to achieve an exquisite and artistic photo in (c) only through a few simple steps. However, The method in (d) utilizes user-specified color strokes to transfer the color style between images. It can be difficult for an amateur to achieve a satisfactory result.

cult to control these strokes. Moreover, these methods do not consider the difference of color spatial distributions between the source image and the target image. Therefore, the output image may lose the visual coherence and some important color characteristics.

In this paper, we propose a new color transfer technique for portrait images by taking advantage of facial semantic information and large-scale Internet images. There are two major contributions in the proposed method. First, we propose a new color transfer framework which transfer colors between local regions of the source image and the target image with the same facial semantic information. Second, we propose a novel automatic trimap generation algorithm for efficient synthesis of each facial semantic information. In order to avoid the time-consuming operation of semantic segmentation for target images, we setup a face database to store the semantic information of target images collected from Internet.

\section{Related Work}

The existing color transfer approaches can be classified into automatic transfer algorithms, interactive methods, and other applications of the color transfer concept. In this section, we review these approaches.

Many automatic transfer algorithms have been proposed that attempt to globally match the color distribution of the original to the reference. Automatic color transfer algorithm was firstly presented by Rein- hard et al. [1]. The pixel color values of the source image are transformed by matching the global color mean and standard deviation of the target in an uncorrelated color space lab. Chang et al. [2] proposed a perceptionbased scheme for transferring colors based on the basic color categories, which are derived through a psychophysical experiments. This framework was extended to stylize the video frame sequences in [3]. L. Neumann and A. Neumann [4] used 3D histogram matching to transfer the color style of a source image into an arbitrary given target image with a different distribution. Tai et al. [5] modeled the image color distribution as Gaussian mixture models and solved the regional color transfer problem by expectation maximization. Piti et al. [6] proposed automated color grading method by transferring an dimensional probability distribution function to another. Dong et al. [7] extracted the same number of dominant colors (DCD) from the two input images and found an optimal one-to-one mapping between the dominant color sets. Pouli and Reinhard [8] presented a histogram reshaping technique which allows users to select how well the color palette of the source image should be matched to that of the target. To further optimize the scene details in the result, Xiao and Ma [9] employed poisson compositing [10] to preserve the gradients of the source image. However, as shown in Fig. 1, the commonly used color transfer algorithms fail to generate a satisfactory result if the number of source dominant colors is much less than the target one, especially when the color styles are transferred between images with similar contents. 
Interactive methods make color transfer more controllable in the photo editing process. Luan et al. [11] introduced a brush by which the user specifies the source and destination image regions for color transfer. Wen et al. [12] used strokes in both the source and target images for multiple local transfers. An and Pellacini [13] used a nonlinear constrained parametric model to transfer colors between image regions defined by pairs of strokes. Liu et al. [14] presented an ellipsoid color mixture map to realize selective color transfers. Compared with those interactive frameworks, our image content structure extraction technology can help to maximize the automation.

The concept of color transfer was extended to other applications. Yang et al. [15] presented a mood-transferring method between color images by using histogram-matching to preserve spatial coherence. Xue et al. [16] tried to transfer the weathering effects between the input images instead of transferring color. Murray et al. [17] modified the color content of an input image given only a concept specified by a user in natural language. Datadriven methods were presented for image color enhancement by quantifying image-color theme difference [18] and non-rigid dense correspondence [19]. Recently Chia et al. [20] proposed a semantic colorization system that leverages the image content on the Internet.

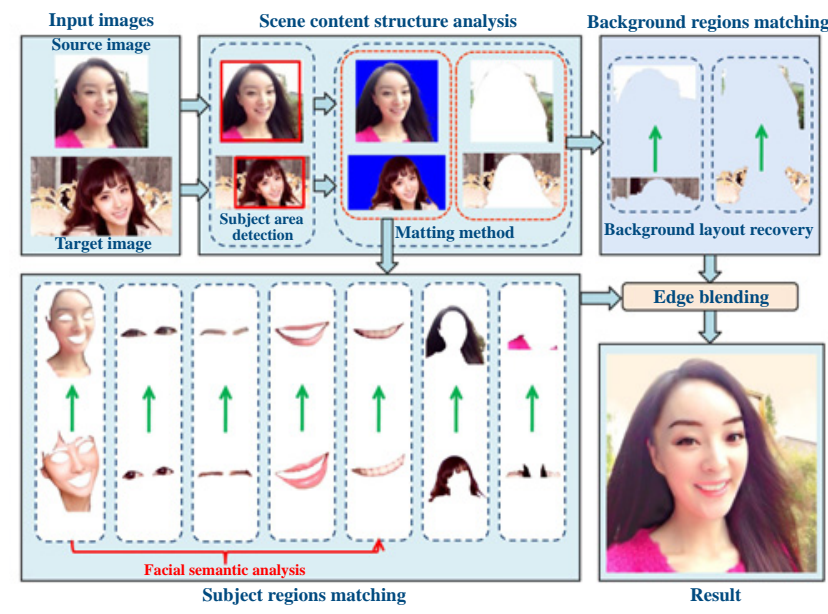

Fig. 3 System pipeline.Color transfer based on scene content analysis and facial semantic analysis. In this system pipeline, the green arrows represent we transfer the color style of the source image according to the target image.

\section{Overview}

In the color style conversion process, we would like to apply the color spatial distribution of the target image to the source image with a good visual coherence between boundaries of each facial semantic information. Therefore, we present a facial semantic information based approach to transfer the color style between images. The system flowchart of our work is shown in Fig. 3. First, we setup a database for target images, and perform the facial feature match for the source image to retrieve a target image from the database (Section 4). Second, in order to improve the process speed and obtain accurate results, we preprocess the target image by using facial semantic segmentation and scene content analysis and save the preprocessing results in the database (Section 5). Third, the facial semantic segmentation and scene content analysis are performed for the source image. Next, we transfer the color style of the target image to the source image based on the corresponding local semantic information. Finally, we can produce the result image by synthesising each semantic region using a simple alpha-blending scheme (Section $6)$.

\section{Face Matching}

\subsection{Face database setup}

Taking into account the fact that using database can not only effectively reduce the redundant data but also be easy to share with other applications. Data security and integrity checking in the database can reduce the problems caused by the data errors. The database can be used by different users simultaneously. Therefore, we use the database to store target images and semantic information of target images, which is useful for putting our research into practice.

The face data should effectively reflect a persons facial features, so we consider many aspects when we establish the face database. The features include the gender, age, race, emotions, facial posture, smile degree, whether wear glasses, whether has a beard and so on. Using these characteristics, we can get a better matching result.

We divide our database into the male group and the female group (Fig. 6) to improve the matching speed. The source image can match the target image in the database quickly and effectively. Finally, we can obtain several target images of greatest similarity with the source image. Therefore, our method is also effective in some mobile devices with limited computing resources.

Meanwhile, we preprocess target images in the database. We mainly use scene content analysis and facial semantic analysis in this process. After preprocessing, we store the semantic information in the database. When we need to transfer color between images, we can use 
the preprocessing results of target images (Fig. 4) to achieve a high speed.

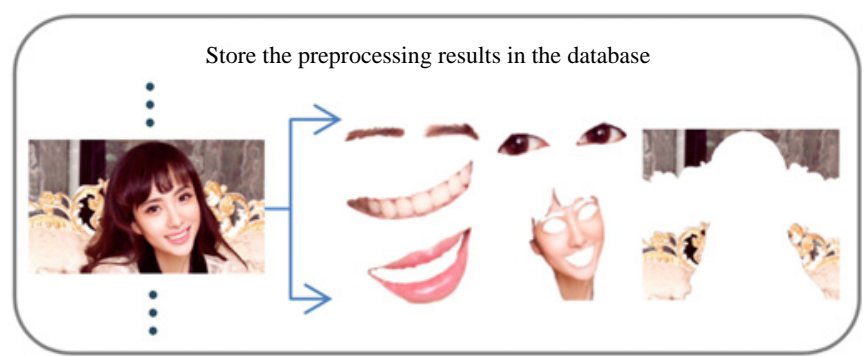

Fig. 4 Store the facial semantic information and the scene structure information in the database after preprocessing the target image.

\subsection{Face matching}

We match the source image and the target image in the target image database, and then obtain the three greatest similarity target images with the source image.

Before the face matching, we need to perform face detection of the source and target images for obtaining the key points of a human face, and obtain the facial characteristics. We can call the API provided by the Face $++[25]$ for face detection. The landmark API can detect the key points of the human face. The API is used to detect the position of the facial contours, facial features and other key points, which include 25points and 83-points modes. In order to improve the sophistication of the face detection, we choose the 83points mode. An example is as illustrated in Fig. 5.

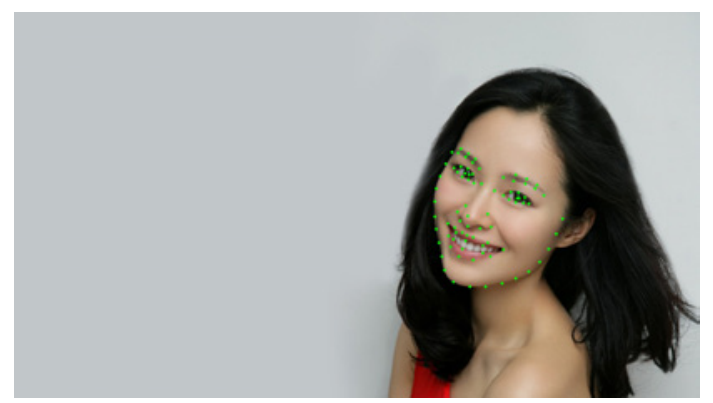

Fig. 5 Our approach detects 83 key points in the face.

After the face detection, we can match the source image and the target image through the 83 key points. First, we need to train faces in the target database. Then, we use the identify API provided by Face++ [25]. When we call this API, the program can calculate the similarity test in the database. After the program finds a certain number of images (we choose 3 images in our system) with greatest similarity to the source image, then it will return the results. The matching process can be represented as Fig. 6 .

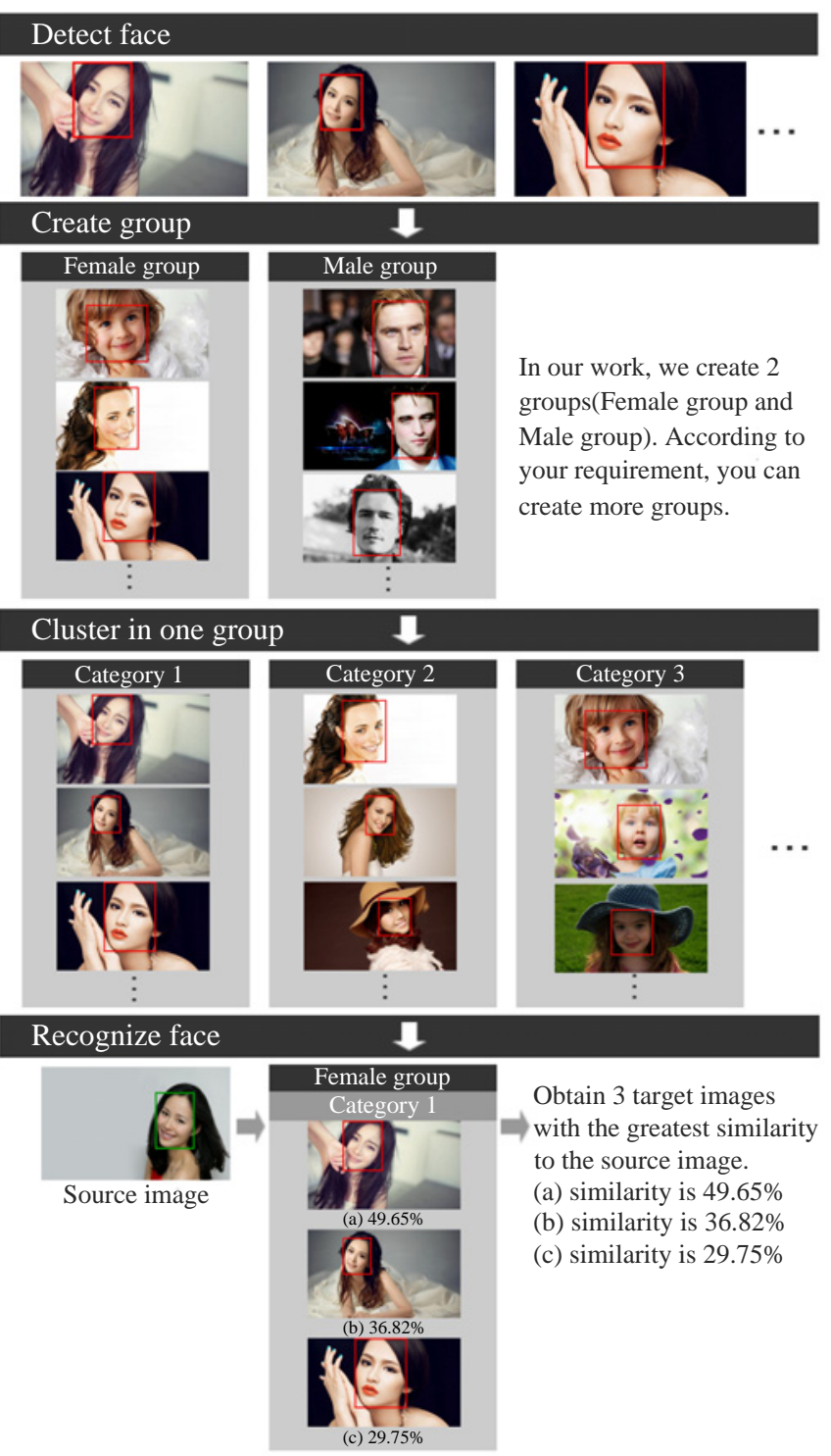

Fig. 6 The flowchart of face matching. Our system suggests three target images whose similarities to the source image are the highest.

In order to help users match the source image and target images in the database quickly and effectively, we design a face match tool based on the Face++ API [25].

There are three steps to use the face matching tool. First, upload the target images to the corresponding group in the database. When uploading images is completed, click to generate the training url. Users can use 
the url in the browser to train images. Finally, users can do the similarity test. Consequently, users can obtain the three target images with the greatest similarity to the source image. The matching results are as shown in the bottom of Fig. 6.

\section{Face Semantic Analysis}

Color transfer based on the face semantic information needs to analyze the facial semantic information of the target and source images respectively. Firstly, we preprocess target images in the database so that avoid the time-consuming operation of facial semantic analysis and scene content analysis for the target image at each color transfer process. Next, in the process of the color transfer, we should preprocess and precisely analyze the facial semantic information and the scene content analysis of the source image, so that we can get the accurate result after color conversion.

\subsection{Scene content analysis}

The analysis of the scene content helps us get a natural and coherent conversion result. In this section, we conduct subject area detection and background layout recovery mainly referring to the method mentioned in [21].

By analyzing the content of the scene, we can separate the portrait from the background, and also get the layout of the background. These operations can reflect the color distribution and spatial distribution of the target image, which is useful for the color transfer. After these operations, we can focus on the human face semantic analysis.

\subsection{Contours of face semantics}

In order to get the outline of the facial semantic information, we can connect the 83 key points on the face based on the face detection.

By calling the landmark API, Face ++ can recognize the facial semantic information using the Json format. In this Json file, the landmark entry stores the key points of the various parts of the human face, including eyebrows, eyes, mouth, chin, face and so on. Each part has some points, and the points are represented by the coordinates using $x$ and $y$. Using these key points, we can connect them in a certain order, then we can get the contour of the face (we set background to black and set outline to white). The face semantic information outline is given in Fig. 7 .
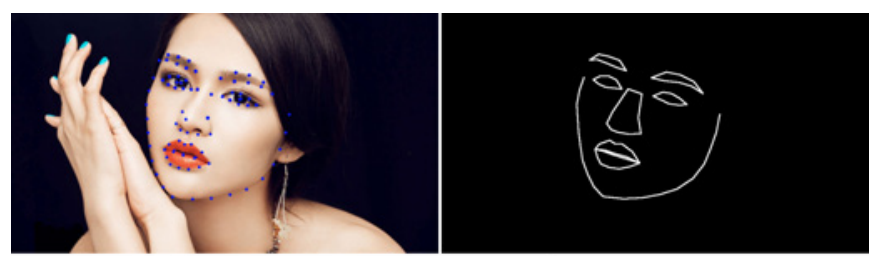

(a) Face detection

(b) Facial semantic information outline

Fig. 7 In a certain order, we connect the 83 key points on the face based on face detection, then the facial semantic information outline can be obtained.

\subsection{Image matting for source and target images}

A commonly used approach to extract semantic information is the Mean-Shift image segmentation technique [22]. However, it will produce unwanted hard boundaries between semantic regions. In this paper, we employ the image matting technique to obtain a semitransparent soft boundaries. Gastal et al. [23] present the first real-time matting technique for natural images and videos. This technique is based on the fundamental observation that pixels in a small neighborhood often have highly similar values for their true $(\alpha, F, B)$ triplets. Therefore, independently treating each pixel in the unknown regions of a TriMap results in a lot of redundant work. They show how this computation can be significantly and safely reduced by means of a careful selection of pairs of background and foreground samples. Here we implement our automatic matting based on their matting technique by taking advantage of our generated TriMap.

Existing static image matting algorithms require through manually tagging to add additional constraints for matting images. TriMap mark is an effective division of a given image, and it can divide the image into the foreground, background, and unknown region. However, constructing a suitable TriMap is very tedious and timeconsuming. Sometimes, the inaccuracy TriMap will lead to a poor matting result. The following figure creates a TriMap of hair using Adobe Knockou2 plug-in which consumes about half an hour.
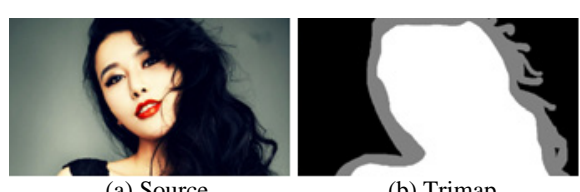

(b) Trimap

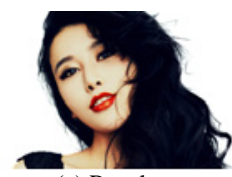

(c) Result

Fig. 8 Create a TriMap using Adobe Knockout plug-in for the hair which consumes about half an hour.

In order to solve the problem mentioned above, we expand the contour of facial semantic information using an expansion algorithm. After distinguishing the fore- 
ground, background and unknown region by different color (we set foreground to white, set background to black, and set unknown region to gray), we can obtain a corresponding TriMap for an image.

The expansion algorithm is to perform convolution for the image (or part of the image, called $A$ ) and its core (called $B$ ). The core can be any shape and any size, and it has a reference point which is defined separately and called an anchor point. The expansion algorithm is used to calculate a local maximum. Core $\mathrm{B}$ performs convolution with image $A$, that is to say, calculate the maximum pixel in the area covered by core $B$ and assign the value to the pixel of the reference point. In this way, the highlighted area in an image (such as the outline in our application) will gradually grow. The principle of the Expansion Algorithm is showed in Fig. 9:

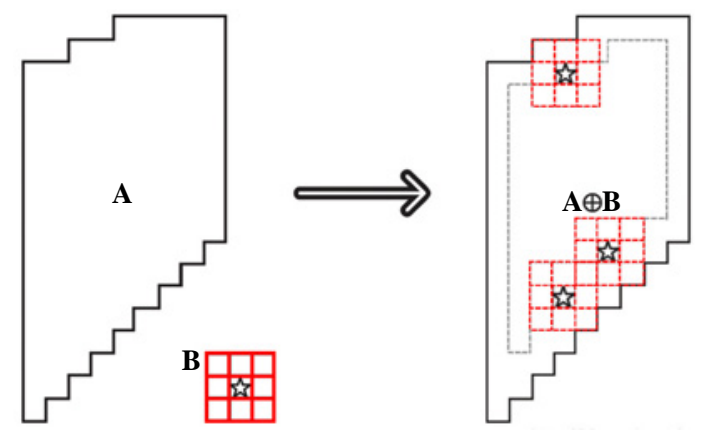

Fig. 9 Principle of the Expansion Algorithm

The mathematical expression for the expansion algorithm is:

$\operatorname{dst}(x, y)=\max _{\left(x^{\prime}, y^{\prime}\right): \text { element }\left(x^{\prime}, y^{\prime}\right) \neq 0} \operatorname{src}\left(x+x^{\prime}, y+y^{\prime}\right)$.

Finally, after the expansion processing we set the contour to gray (represent the unknown region), foreground color to white, and background color to black, we can obtain a corresponding TriMap for an image. The Trimap result is shown in Fig. 10. Consequently, the matting image is computed with our automatically generated Trimap [23]. The transparent image and the synthetic image are shown in Fig. 11:

The automatic matting approach is also applied in source images to obtain the basic semantic segmentation. In order to achieve a more exquisite effect, we develop a stroke-based matting system by solving a quadratic energy function and provide users with an interactive tool for image matting. This tool can help users enhance the effect of matting.

As shown in Fig. 12, an energy minimization framework for interactive image matting is proposed by Guan

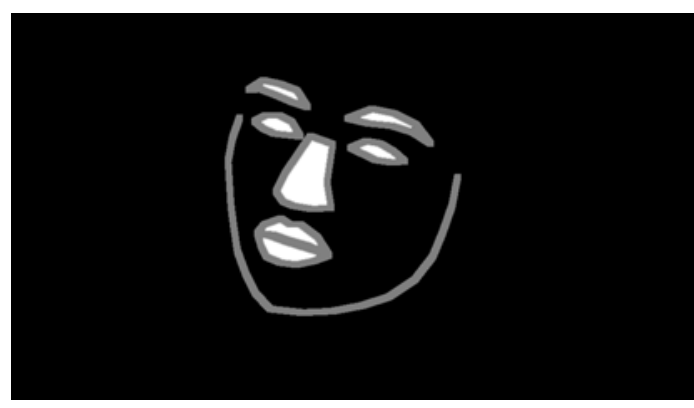

Fig. 10 TriMap for the target image

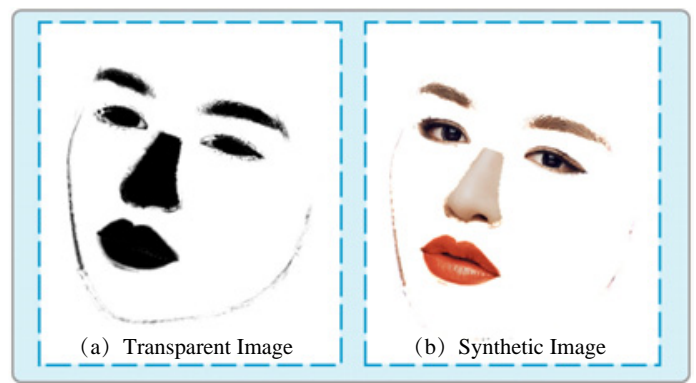

Fig. 11 Transparent image and synthetic image.

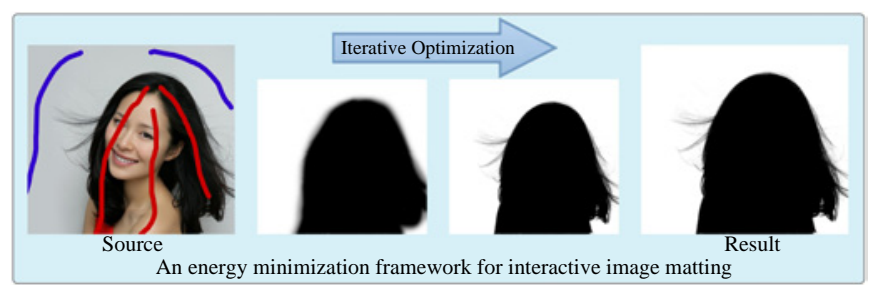

Fig. 12 The red strokes represent foreground, the blue strokes represent background.

et al. [24]. Users start from a few strokes, and iteratively estimate the matte until it converges.

Beginning with the known region, we model the unknown region as a Markov Random Field (MRF) and formulate its energy $E$ below in each iteration as the combination of one data term and one smoothness term as in [24]:

$E=\sum_{p \in \Omega}\left(E_{d}(p)+\lambda E_{s}(p)\right)$

where $p$ represents a pixel. $E_{d}$ is the data energy which describes how well the estimated alpha value $\alpha_{p}$, the foreground $F_{p}$ and background $B_{p}$ fit with the actual color $C_{p} . E_{s}$ is the smoothness energy which penalizes the inconsistent matte changes between $p$ and its neighborhood $N(p) . \lambda$ is a dynamically adjusting parameter which is used to balance the influences of the data term and the smoothness term.

By automatically adjusting the weights of both terms during the iterations, the first-order continuous and 
feature-preserving result is rapidly obtained with several iterations. The energy optimization can be further performed in selected local regions for refined results.

Based on this method, we developed a stroke-based matting tool as shown in Fig. 12. This tool is easy in the sense that it is fast and requires only few user-specified strokes to mark the foreground and background. Furthermore, it can help users enhance the effect of matting to achieve sophisticated processing for source images.

\section{Color Transfer}

In this section, we transfer the color style between corresponding semantic information of the source image and the target image.

In its simplest form, the algorithm proposed by Reinhard et al. [1] shifts and scales the pixel values of the source image to match the mean and standard deviation of the target in $L \alpha \beta$ color space. Change the color space from $R G B$ to $L \alpha \beta$, because the three components $R, G, B$ are highly related, it is difficult to adjust each channel individually.

$L_{o}=\frac{\sigma_{t, l}}{\sigma_{s, l}}\left(L_{s}-\mu_{s}\right)+\mu_{t}$

Here, the subscripts $s, t$ and $o$ correspond to the source, target and output images, $\mu$ and $\sigma$ are their respective means and standard deviations. Equation 3 describes the distribution transfer between source and target for the luminance channel only. The same process is repeated for the two opponent channels of the $L \alpha \beta$ space to complete the color transfer.

After each semantic region are color transferred, we employ the simple alpha-blending scheme to composite the whole portrait image. The alpha channel of each pixel is computed with the aforementioned image matting technique. Fig. 13 shows the processing of color transfer between the corresponding semantic information of the source image and the target image.

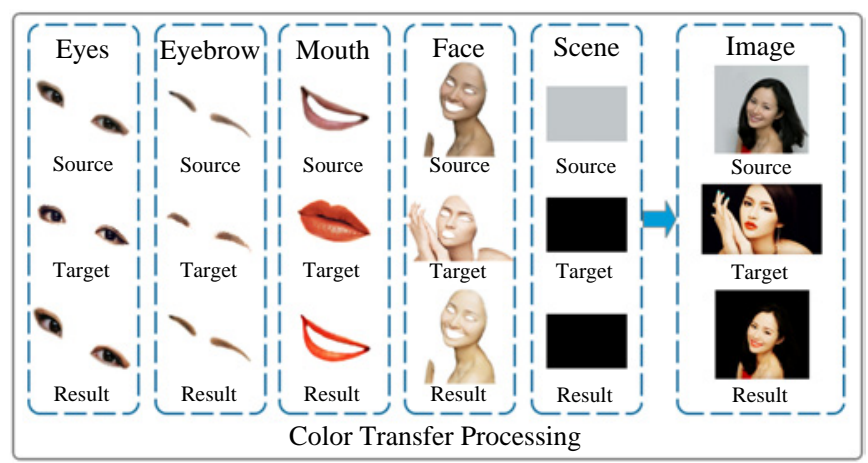

Fig. 13 Color transfer processing

\section{Result and Discussion}

We proposed a method based on facial semantic information to convert the color style of images. We implemented our method on a machine with an Intel Core (TM) i5-3470 3.20GHz processor and 4GB RAM. The amount of time of a target image preprocess is typically about $20 \sim 30$ seconds in total (the target images from $1920 \times 1080$ to $1125 \times 1080$ ). The processing of source image semantic analysis by using the interactive tool achieves real-time performance. The amount of time of using our tool to match a source image in the target image database is showed in Table 1.

The result in Fig. 14 shows a typical example that benefits from our method. Our approach retains the contents of the source image and gains the spatial distribution of the target image, it also has the strong visual coherence.

Figure 14 shows the comparison between our method and the approach of Reinhard et al. [1]. We find if there are the following kinds of situations in target images, it will lead to a bad result: (1). Color in different regions has great differences. (2). Color in a source image is much less than color in a target image. (3). A target image has high saturation hue and high-frequency hue. Because the color transfer algorithm in [1] adopts the method for calculating the mean and variance, so the color spatial distribution in a target image is mean to each pixel of each channel in a source image. In contrast, our method transfers color style between the corresponding semantic information, so that we can gain the spatial distribution of the target image and keep the strong visual coherence.

Figure 15 shows the comparison between our method and the approach by $\mathrm{Wu}$ et al. [21]. We find if there are the following situations in the target image, it will lead to a bad result: (1). Color in different regions has great differences. (2). Color in a source image is much less than color in a target image. (3). A target image has high saturation hue and high-frequency hue. Because the color transfer algorithm in [21] adopts the method for calculating the mean and variance, so the color spatial distribution in a target image is mean to each pixel of each channel in a source image. In contrast, our method transfers color style between the corresponding semantic information, so that we can gain the spatial distribution of the target image and keep the strong visual coherence.

Figure 16 shows the color transfer method of [13]. This paper presents an image editing framework where users use reference images to indicate desired color edits. The interactive method utilizes user-specified color strokes. However, it can be difficult for an amateur to 
Table 1 Statistical Results of Face Matching

\begin{tabular}{c|c|c|c|c}
\hline Image Size(px) & Target Image Size(px) & Target Image Number & Processing Time(s) & Highest similarity \\
\hline $1920 \times 1080$ & $1125 \times 1080 \sim 1920 \times 1080$ & Female group: 200 & $8 \sim 10$ & $60.63 \%$ \\
\hline $1920 \times 1080$ & $1125 \times 1080 \sim 1920 \times 1080$ & male group: 200 & $8 \sim 10$ & $57.15 \%$ \\
\hline $1920 \times 1080$ & $1125 \times 1080 \sim 1920 \times 1080$ & Female group: 500 & $12 \sim 17$ & $63.57 \%$ \\
\hline $1920 \times 1080$ & $1125 \times 1080 \sim 1920 \times 1080$ & male group: 500 & $11 \sim 15$ & $59.32 \%$ \\
\hline $1920 \times 1080$ & $1125 \times 1080 \sim 1920 \times 1080$ & Female group: 1000 & $18 \sim 23$ & $76.63 \%$ \\
\hline $1920 \times 1080$ & $1125 \times 1080 \sim 1920 \times 1080$ & male group: 1000 & $17 \sim 22$ & $76.63 \%$ \\
\hline $1920 \times 1080$ & $1125 \times 1080 \sim 1920 \times 1080$ & Female group: 2000 & $30 \sim 36$ & $80.57 \%$ \\
\hline $1920 \times 1080$ & $1125 \times 1080 \sim 1920 \times 1080$ & male group: 2000 & $28 \sim 34$ & $79.32 \%$ \\
\hline
\end{tabular}

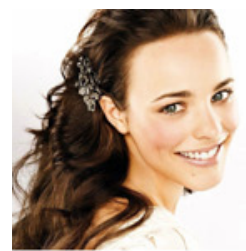

(a) Source Image

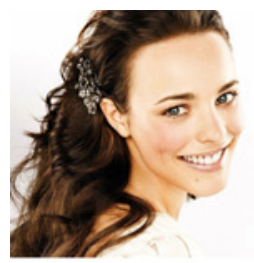

(a) Source Image

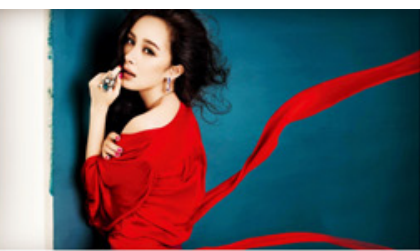

(b) Target Image

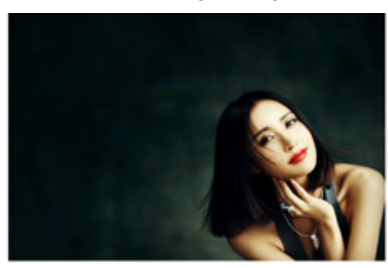

(b) Target Image

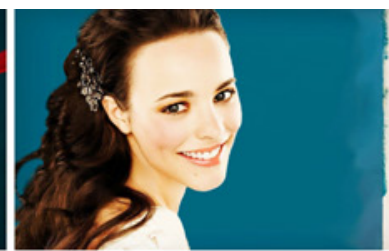

(c) Our Result

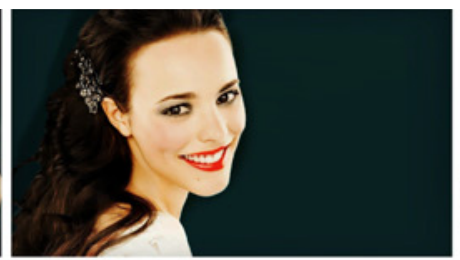

(c) Our Result

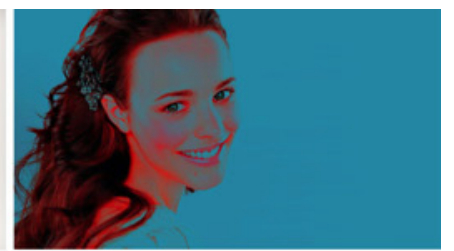

(d) [RAGS01]

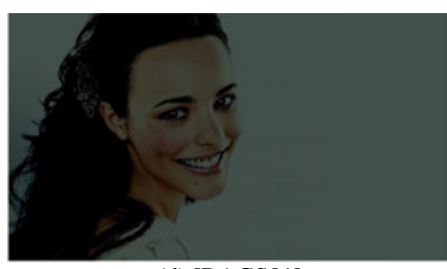

(d) [RAGS01]

Fig. 14 comparison between our method and [RAGS01]

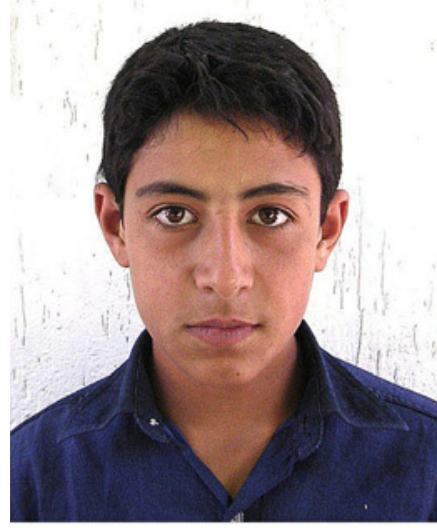

(a) Source Image

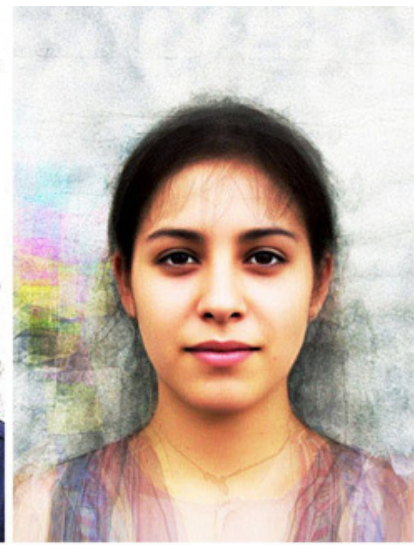

(b) Target Image
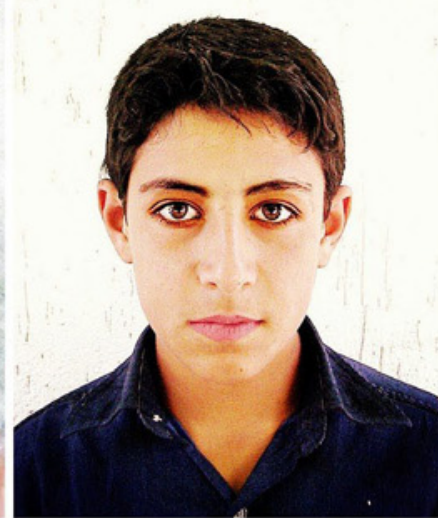

(c) Our Result

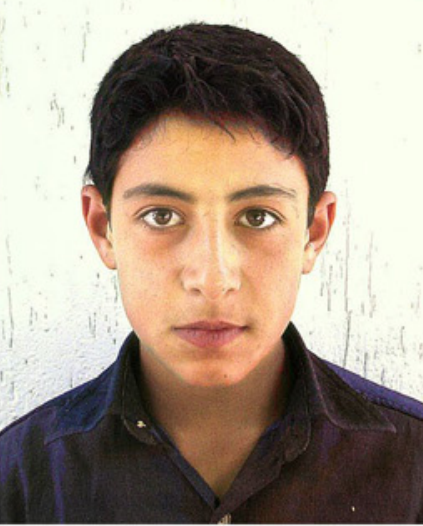

(d) [WDZ13]

Fig. 15 comparison between our method and [WDZ13]

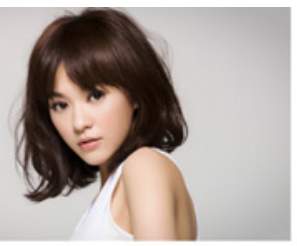

(a) Source Image

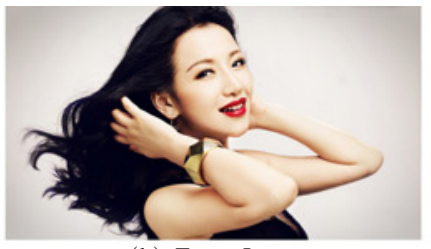

(b) Target Image

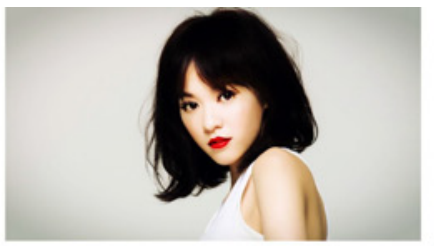

(c) Our Result

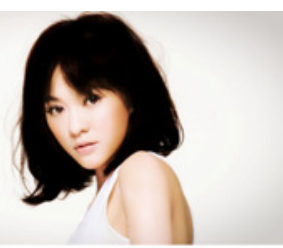

(d) $[\mathrm{AP} 10]$

Fig. 16 comparison between our method and [AP10] 

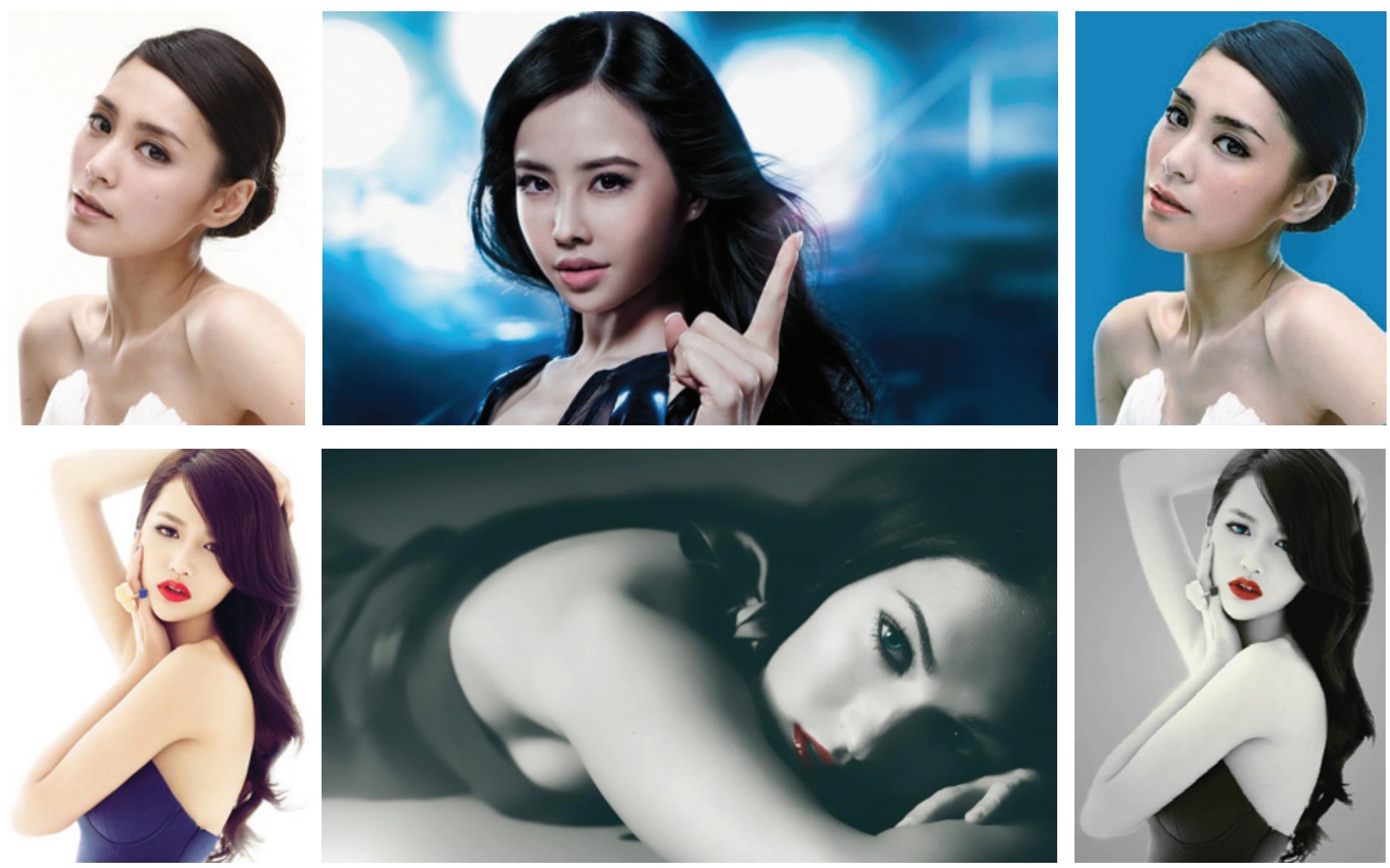

Fig. 17 More color transfer results

specify those strokes in a compatible and perceptually coherent manner. In our method, through facial semantic analysis and some processing, we can get the corresponding TriMap. By using TriMap, image matting achieves automation.

More portrait color transfer examples are presented in Fig. 17. In each row, the left image is the source, the middle one is the target, and the right one is our result. From the example of the second row in Fig. 17, we can find that our approach can deal with examples even some parts of them are in grayscale.

\section{Conclusion and Future work}

In our paper, we have proposed a new portrait image color transfer framework to efficiently convert the color style of an image. With a better understanding of high level facial semantic information and scene content, our method well preserves the spatial layout, the color distribution and the visual coherence in the transfer process with minimal manual labor.

This work has the following benefits:

Strong practicability: Our work can help amateurs who are lack of time and expertise to transfer a common portraiture to achieve an exquisite and artistic photo through a few simple steps.
Good effect: To accurately transfer colors between images with minimal manual labor, we present a system which benefits from the high level facial semantic analysis and scene content analysis. Experiments show that our method has a good effect on image color conversion, especially for portraiture.

High performance: In our method, we preprocess the target image in the database so that we can guarantee the process speed, and avoid the time-consuming operation of semantic segmentation for the target image.

Broad application prospect: Our method can be widely used in film post-production, video-editing, art and design and image processing. As a necessary extension, our method can also be used to re-color video clips with spatially-varied color effects.

In the future, we will extend our method to process the video clip to meet the demand for video color transfer.

\section{References}

1. Reinhard E, Ashikhmin M, Gooch B, et al. Color transfer between images[J]. IEEE Computer graphics and applications, 21(5), 34-41(2001).

2. Chang Y, Saito S, Uchikawa K, et al. Example-based color stylization of images[J]. ACM Transactions on Applied Perception, 2(3), 322-345(2006). 
3. Chang Y, Saito S, Nakajima M. Example-based color transformation of image and video using basic color categories[J]. Image Processing, IEEE Transactions on, 16(2), 329-336(2007).

4. Neumann L, Neumann A. Color style transfer techniques using hue, lightness and saturation histogram matching $[\mathrm{C}] / /$ Proceedings of the First Eurographics conference on Computational Aesthetics in Graphics, Visualization and Imaging. Eurographics Association, 111-122(2005).

5. Tai Y W, Jia J, Tang C K. Local color transfer via probabilistic segmentation by expectationmaximization $[\mathrm{C}] / /$ Computer Vision and Pattern Recognition, 2005. CVPR 2005. IEEE Computer Society Conference on. IEEE, 1, 747-754(2005).

6. Piti F, Kokaram A C, Dahyot R. Automated colour grading using colour distribution transfer[J]. Computer Vision and Image Understanding, 107(1), 123-137(2007).

7. Dong W, Bao G, Zhang X, et al. Fast local color transfer via dominant colors mapping[C]//ACM SIGGRAPH ASIA 2010 Sketches. ACM, 46(2010).

8. Pouli T, Reinhard E. Progressive color transfer for images of arbitrary dynamic range[J]. Computers and Graphics, 35(1), 67-80(2011).

9. Xiao X, Ma L. Gradient-Preserving Color Transfer $[\mathrm{C}] / /$ Computer Graphics Forum. Blackwell Publishing Ltd, 28(7), 1879-1886(2009).

10. Prez P, Gangnet M, Blake A. Poisson image editing $[\mathrm{C}] / / \mathrm{ACM}$ Transactions on Graphics (TOG). ACM, $22(3), 313-318(2003)$.

11. Luan Q, Wen F, Xu Y Q. Color Transfer Brush $[\mathrm{C}] / /$ Pacific Conference on Computer Graphics and Applications. 465-468(2007).

12. Wen C L, Hsieh C H, Chen B Y, et al. Example-based Multiple Local Color Transfer by Strokes[C]//Computer Graphics Forum. Blackwell Publishing Ltd, 27(7), 17651772(2008).

13. An X, Pellacini F. User-Controllable Color Transfer[C]//Computer Graphics Forum. Blackwell Publishing Ltd, 29(2), 263-271(2010).

14. Liu S, Sun H, Zhang X. Selective color transferring via ellipsoid color mixture map [J]. Journal of Visual Communication and Image Representation, 23(1), 173-181(2012).

15. Yang C K, Peng L K. Automatic mood-transferring between color images[J]. Computer Graphics and Applications, IEEE, 28(2), 52-61(2008).

16. Xuey S, Wang J, Tong X, et al. Image-based Material Weathering $[\mathrm{C}] / /$ Computer Graphics Forum. Blackwell Publishing Ltd, 27(2), 617-626(2008).

17. Murray N, Skaff S, Marchesotti L, et al. Towards automatic concept transfer[C]//Proceedings of the ACM SIGGRAPH/Eurographics Symposium on Non-Photorealistic Animation and Rendering. ACM, 167-176(2011).

18. Wang $\mathrm{B}, \mathrm{Yu} \mathrm{Y}$, Wong $\mathrm{T} \mathrm{T}$, et al. Data-driven image color theme enhancement [J]. ACM Transactions on Graphics (TOG), 29(6), 146(2010).

19. HaCohen Y, Shechtman E, Goldman D B, et al. Non-rigid dense correspondence with applications for image enhancement $[\mathrm{C}] / / \mathrm{ACM}$ Transactions on Graphics (TOG). ACM, 30(4), 70(2011).

20. Chia A Y S, Zhuo S, Gupta R K, et al. Semantic colorization with internet images[J]. ACM Transactions on Graphics (TOG), 30(6), 156(2011).

21. Wu F, Dong W, Kong Y, et al. Content-Based Colour Transfer $[\mathrm{C}] / /$ Computer Graphics Forum. Blackwell Publishing Ltd, 32(1), 190-203(2013).

22. Comaniciu D, Meer P. Mean shift: A robust approach toward feature space analysis[J]. IEEE Transactions on
Pattern Analysis and Machine Intelligence, 24(5): 603-619 (2002).

23. Gastal E S L, Oliveira M M. Shared Sampling for Real-Time Alpha Matting[C]//Computer Graphics Forum. Blackwell Publishing Ltd, 29(2), 575-584(2010).

24. Guan Y, Chen W, Liang X, et al. Easy MattingA Stroke Based Approach for Continuous Image Matting $[\mathrm{C}] / /$ Computer Graphics Forum. Blackwell Publishing, Inc, 2006, 25(3): 567-576.

25. http://www.faceplusplus.com.cn/ 